CLAWAR 2020: $23^{\text {rd }}$ International Conference on

Climbing and Walking Robots and the Support

Technologies for Mobile Machines,

Moscow, Russian Federation, 24-26 August 2020.

https://doi.org/10.13180/clawar.2020.24-26.08.39

\title{
AUTOMATED INSPECTION OF SUBSEA STRUCTURE: A CASE STUDY
}

\author{
A. KAUR and R. ANVO \\ London South Bank Innovation Centre, \\ Granta Park, Great Abington Cambridge, UK \\ E-mail: kaura13@lsbu.ac.uk,anvon@lsbu.ac.uk \\ M. O. TOKHI \\ School of Engineering, London South Bank University, London, UK \\ E-mail:tokhim@lsbu.ac.uk
}

\begin{abstract}
The extreme operational environmental conditions and aging conditions of subsea structures pose risk to their structural integrity and is critical to their safety. Non-destructive testing plays a key role in identifying defects developing within the structure, allowing repair in a timely manner to mitigate against failures that cause damage to the environment and pose a hazard to human operators. However, in order to be cost effective, inspections must be carried out in-situ and this poses significant safety risks if undertaken manually. This paper presents the development of an automated inspection system of flexible risers that are used to connect wellheads on the seafloor to the offshore production and storage facility. Due to the complex structure of risers, radiography is considered as the technique suitable to inspect multiple layers of the risers. However, radiography inspection in turn requires a robotic system for in-situ inspection with higher payload capacity, precise movement of source and detector and withstand extreme operational environment. The deployment of the inspection system has been achieved by implementation of a bespoke robotic scanning system that can accurately control the source and detector motion. The prototype was mounted on a flexible riser during shallow water sea trials. The results from the trial show that the internal inner and outer tensile armour layer in the riser could be successfully imaged in real operational conditions.
\end{abstract}

\section{Introduction}

Flexible pipeline technology was pioneered in the 1970s and used initially in benign, shallow water conditions such as the Mediterranean. They began to be adopted by industry during the 1980s as the technology developed and matured to the point where they have now become a key enabling technology to provide access to fields in extreme environments. They have been widely adopted for connecting floating equipment (such as FPSOs [Floating Production, Storage and Offloading]) to the seabed because their inherent flexibility overcomes the challenges posed by dynamic environments [1]. Flexible risers are a type of pipework that connect offshore platforms to sub-sea equipment for production and drilling purposes, and can carry a range of fluids such as hydrocarbons, injection and control fluids and gas lift. There are several types of riser widely used by the oil and gas industry, including steel catenary and top tensioned, that are constructed from stiff carbon steel pipe. This research, however, focuses on flexible risers. The demand for flexible risers is growing; currently the global market is equivalent to around $1,200 \mathrm{~km} /$ year of new pipe [2].

The riser's inherent flexibility is achieved by employing a number of layers in the construction of the riser wall as shown in Figure 1. These layers are able to slip past each other, thereby imparting low bending stiffness characteristics. Each layer has a particular function that is configured by the designer. The inner steel carcass acts to prevent collapse of the liner under hydrostatic pressure and prevent fluid pressure leaking into the annulus. Around this, a polymer sheath provides the seal preventing leakage of the bore fluid passing into the annulus. There are 
several layers of steel wound armour; the pressure armour resists radial hoop stresses and the tensile armour carries the weight of the pipe by resisting axial tension. There can be multiple layers required in high tension applications. The outer polymer sheath is a barrier to sea water but is susceptible to accidental damage. All flexible pipes are designed based on this concept, even if there are variations in material selection for specific operating environments such as high temperature or deep water.

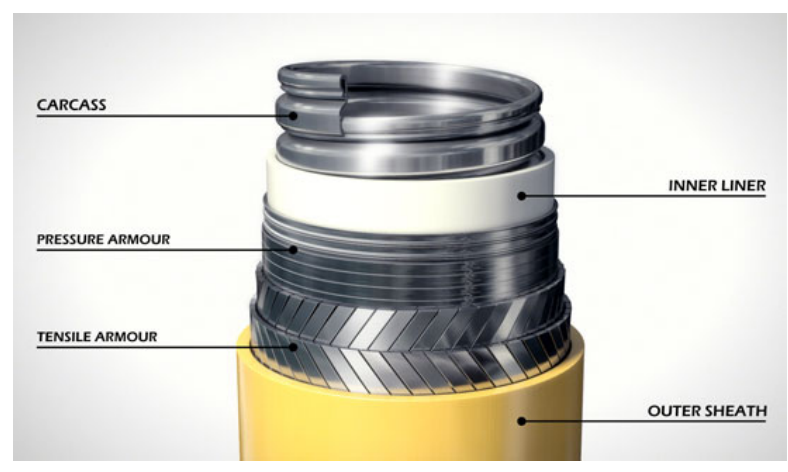

Figure 1. Riser construction [3].

Section 2 of this paper describes the most common flexible riser failure modes and flaws detection. Section 3 describes robotic system prototype development. Section 4 describes the system deployment for subsea flexible risers' inspection. Section 5 presents conclusions and future work.

\section{Flexible risers flaws detection}

Presently, it is difficult for flexible risers to meet the industry's equipment life expectations of 25 years, which steel risers can achieve, due to in-service failure. There are a wide range of potential failure mechanisms detailed in the American Petroleum Institute (API) Recommended Practise guide [4].

The most commonly reported failures in flexible risers relate to damage to the external sheath which lead on to other problems such as corrosion. Sheath damage can occur due to accidents during installation, in-service abrasion with other parts of the installation or polymer aging [5]. Examples of damaged risers are shown in Figure 2.

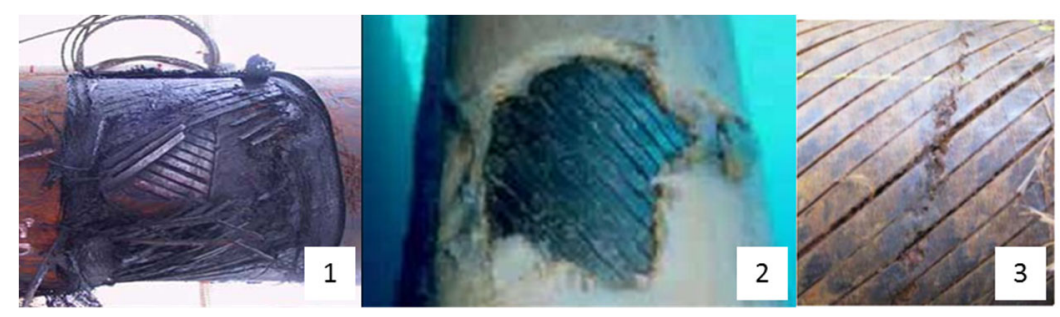

Figure 2. Riser damage: 1) armour wire breakage, 2) external sheath damage, 3) armour corrosion.

There is a drive within industry to better understand riser degradation and failure mechanisms. For example, the Petroleum Safety Authority (PSA) commissioned a 2014 study to focus on degradation, failure modes, inspection and integrity management [6]. Recent Norwegian data indicates that there is at least a $1.5 \%$ probability of riser failure per year in service. As a result, few risers have met their documented service life.

Riser integrity management is a continuous assessment process applied throughout design, 
construction, installation, operations and decommissioning phases to assure that risers are managed safely. As for in-service integrity, the DNV standards require that design intent is maintained, and the actual state of riser degradation is known, due to operational conditions [7]. Part of the degradation assessment requires periodic asset inspection to be carried out.

Only radiography can offer the capability for volumetric inspection to detect flaws in the areas of most concern. However, traditional radiography using wet film or phosphor plates is not in widespread use in the subsea environment because it is impractical and time consuming, and therefore expensive to execute. This is because, after exposure of the radiography detection media, it is then necessary for the media to be returned topside to be developed and processed [8][9]. The application of digital radiography (DR), whereby a marinised underwater flat panel detector is deployed as the radiography detection media, has been previously researched and demonstrated both by the current authors [10] and subsequently by others [11] in later work.

DR allows for fast acquisition of radiographic images through shorter exposure times and fast transfer of radiographic image data. It is not necessary to return the radiography detection media to the surface in-between each radiographic exposure as the recovery of the radiographic images is achieved through digital communication, via an umbilical, to a remote computer located topside. However, for the widespread uptake of DR in the oil and gas industry, DR deployment needs to be further automated and more cost effective.

\section{Robotic system prototype development}

The development aim of the robotic system is to facilitate in-situ inspection of the flexible riser without the need to remove it from service. Risers are designed to operate in the oil field for 25 or more years, and therefore require periodic inspection to guarantee their integrity. As removing them from service is prohibitively expensive, RiserSure must be able to deliver high quality inspection data in the field. The conventional methods of deploying inspection systems around a riser are either by diver or remotely operated vehicle (ROV). The intervention of a diver comes with associated operational risks and, particularly in the case of radiography inspection, is not easy as the inspection units are relatively heavy compared to other inspection techniques. The radiography source and detector need to be aligned directly opposite to each other and moved very precisely at very low speed. The option of deploying the inspection system around the riser using a ROV is not favoured on cost grounds. Costs associated with work class ROVs are very high depending on their payload capacity and the time required for operation.

The robotic system has been designed such that divers and ROVs are not necessary. Therefore, the robot capsule can be connected to the riser near a top side hang-off point, and winched down the riser (as shown in Figure 3). This allows inspection in the critical areas where riser damage often occurs; the splash zone down to $50-100 \mathrm{~m}$ below the surface.

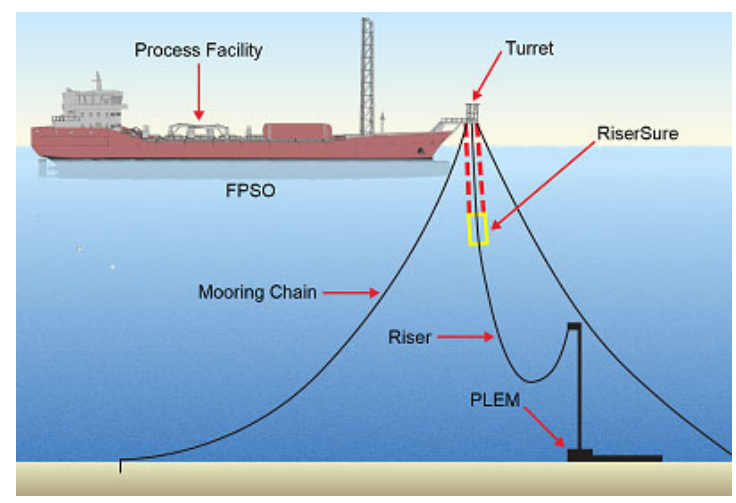

Figure 3. Typical deployment strategy for the RiserSure capsule. 
The main operational requirements of the robotic system include its capability to carry the radiography unit to the point of inspection, securely hold its position around the riser, and complete a $360^{\circ}$ scan of the riser. The system has the capability to vary the scanning speed to change the exposure time such that it can accommodate a wide range of riser diameters. Specifically, it can manage risers of up to $250 \mathrm{~mm}$ (the majority of risers are of $250 \mathrm{~mm}$ diameter or less in the North Sea [5]). In order to build up a detailed radiographic image that will capture small defects, the rotational speed of the drive system on which the radiography system is mounted is very low and precisely controlled.

The core of the prototype robotic system is an open platform that can be positioned around a riser with very little or no manual adjustment. The only part of the capsule that needs to be opened to encircle a riser is the two piece precision rotary ring gear that is used to carry the radiography units. The design approach is such that it can easily be adapted to be deployed using ROVs if desired. The rotary ring is actuated by a motor driven pinion gear, and floats on three thrust bearings fixed permanently on a base plate. A model of the prototype is shown in Figure 4. To keep the radiography units stable against the wave disturbances during scanning, and to avoid any collisions with the riser surface, a gripper system consisting of linear slides has been incorporated which holds the riser during scanning inspection. The system has been designed to work up to $100 \mathrm{~m}$ of depth in subsea environment.

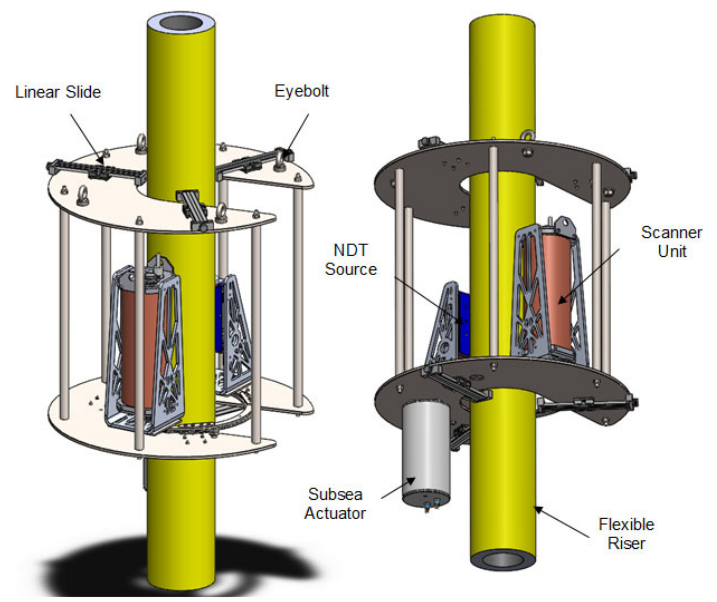

Figure 4. 3D model of prototype of the robotic inspection system.

The gripping and scanning system of the riser has been implemented using customised subsea actuators. Each of the actuators has the drive and control circuitry integrated within the subsea enclosures. Each actuator includes position feedback, current limit control, internal temperature and status monitoring registers. The low level controls are implemented in the integrated controller, and the supervisory control of the whole system is implemented in a dedicated industrial computer which is operated remotely at the topside. All the robotic subsea units are connected to the topside unit using the controller area network (CAN) bus interface. Each actuator acts as a node with its own unique ID. All actuators are connected to the topside unit via a single integrated umbilical. This ensures minimal wiring required for interconnections and is key to good cable management practice. The umbilical contains a pair of cores for power supply and a twisted pair of cores for communication to the topside controller. This reduced size and weight of the umbilical helps in reducing drag on the whole robotic system in the subsea conditions, while at the same time providing benefits of CAN bus such as communication error control, bus arbitration and noise immunity. 
There are high costs involved for fabrication of subsea enclosures. The robotic electronics hardware configuration has been designed specifically to minimise the size of enclosure units and junction boxes. This is achieved by using dedicated in-built control and drive units of the actuators which are interconnected using a subsea umbilical with splice extensions and subsea connectors.

The system configuration of the robotic inspection system, consisting of robotic hardware and the radiographic modules, is shown in Figure 5. The inspection detector consists of the in-built read-out and processing circuitry. The array detector outputs are transferred to the topside dedicated processing software via an Ethernet interface. The digital detector has a separate DC power supply and controller, which is supplied with the system. The source controller unit contains the hydraulic supply and valve system, which controls the subsea source unit to open or close the shutter for exposure.

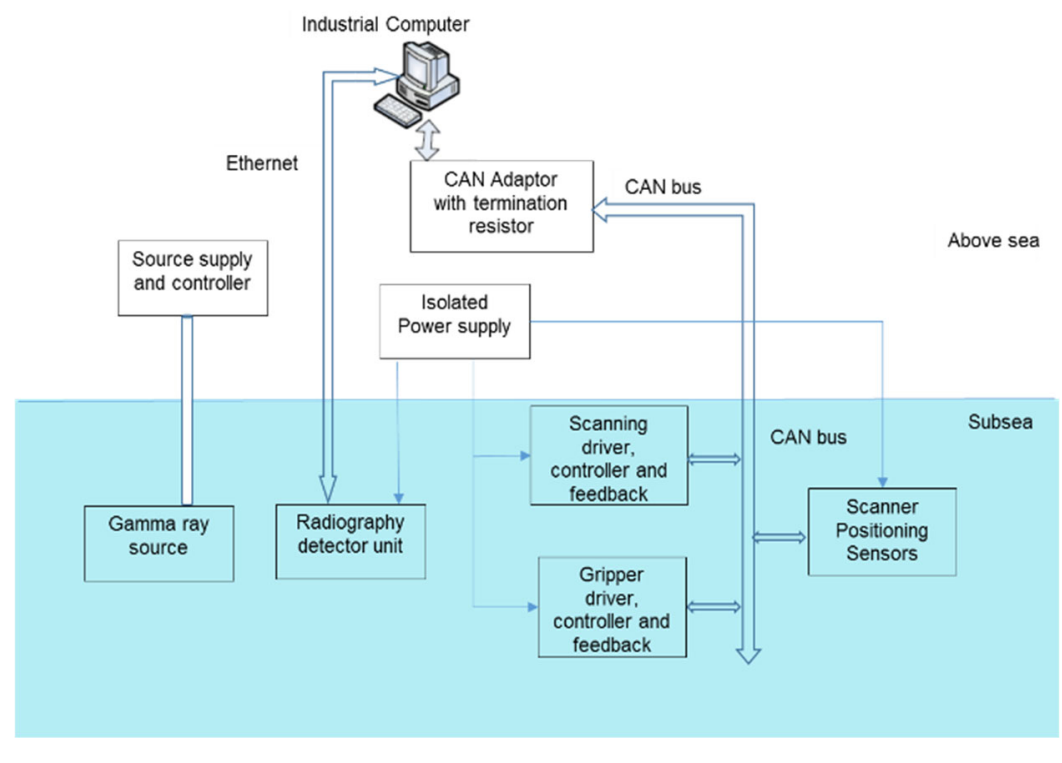

Figure 5. Robotic inspection system configuration.

The supervisory control on the topside is a GUI (graphical user interface) based system. This includes the control of system operational modes based on user inputs and internal system parameters, monitoring of critical parameters of subsea units, display of system status and warnings of system errors. The guidelines from subsea standards are implemented in the control software. This consists of the timing parameters for sampling of inputs, display updating rate, continuous position monitoring of the robotic system, display of modes being executed, checks on user inputs, provision for stopping execution for any of the operational modes and saving of data as required. The hardware and software of the robotic system are based on a modular structure that facilitates easy integration of additional hardware units in the system and respective software components. The winch system is a subsea certified commercially available system which is operated independently. However, for safety purposes, before the execution of any operational modes, except the emergency stop, the user is required to confirm the operation, allowing the verification of external and internal systems' conditions. To resist the subsea environment and to withstand the environmental loading, all the mechanical parts and enclosures are manufactured from stainless steel.

\section{Robotic deployment for subsea flexible risers inspection}

Prior to the seawater trials, the RiserSure system had been subjected to several laboratory based trials which demonstrated that the system had the capability to transition to seawater operation. 
The shallow water trials $(<10 \mathrm{~m}$ depth) were performed at the seawater-fed Loch Linnhe in Fort William. By having this first trial at a sheltered sea loch first, before actual sea trials on an FPSO, meant that it was possible to validate the RiserSure system operation in a more controlled environment. The RiserSure system was managed from the control room on the surface through an umbilical link, allowing the system to remotely image a full $360^{\circ}$ section of the riser. A photograph of the submerged system is shown in Figure 6 where the core functionality of the system was proven. Due to the shallow depth $(<10 \mathrm{~m})$ the source container could be pneumatically operated using compressed air. High quality, radiographic images of the riser were captured and tested for different exposures and varying scanning speeds. A key factor in obtaining a high quality radiographic image with the RiserSure system is the synchronisation between the detector's image acquisition speed and the rotational speed of the scanner module. For optimal operation, the radiography detector required to acquire a line of the image each time the object moved a distance equal to the detector's pixel width (assuming no geometric magnification of the radiographic image). If the detector acquires the line images too fast then the resultant radiograph becomes stretched, a too slow acquisition results in a compressed image, which could lead to image blur and small defects being masked and undetectable.

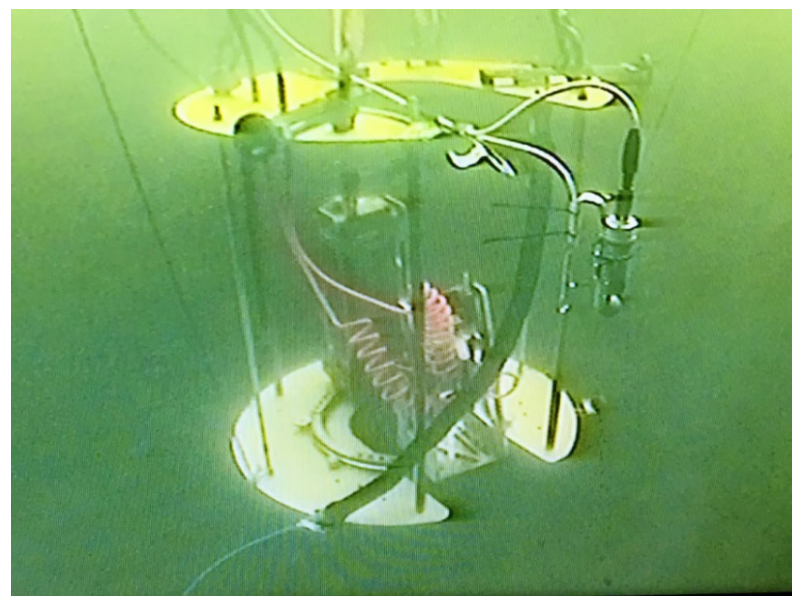

Figure 6. Robotic inspection system configuration.

Figure 7 shows a radiographic image for a $360^{\circ}$ scan of the riser which resulted in two impressions of the artificially created defects in the acquired image. One is relatively, proportional in size at low magnification when the detector passes the defect side, and the other is a highly magnified but distorted version where the Gamma source passes the defect side.

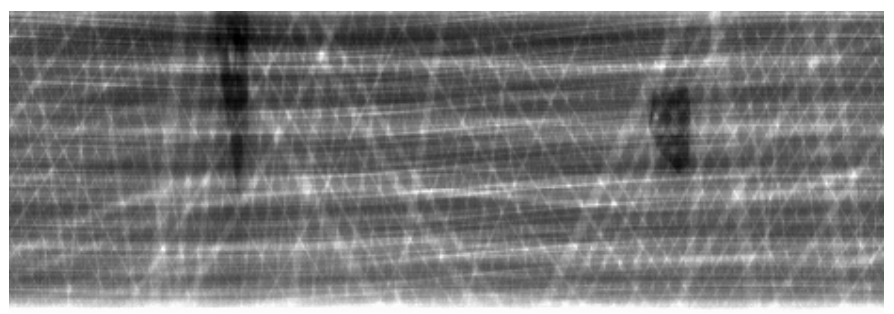

Figure 7. Radiographic image showing $360^{\circ}$ scan.

The results achieved during trials revealed the riser's internal structure, proving the robotic inspection system's suitability to carry out radiographic inspection of the flexible riser and providing reliable images of high accuracy and resolution. 


\section{Conclusion}

The developed robotic system has been demonstrated and proven it's capability to carry out inspection of flexible risers using radiography in the operational conditions. With the digital radiography method, it is possible to visualize the radiography results in real-time.

\section{Acknowledgments}

Authors acknowledge the European Commission that funded the project RiserSure and provided the opportunity to carry out the presented work.

\section{References}

1. J. Remery, C. Silva, O. Mesnage, "'The free standing flexible riser: A novel riser system for an optimised installation process", Offshore Technology Conference 2008, Houston

2. G. Gardner, P. Viana, "Flexible Riser Re-use and Life Extension", AOG 2016

3. Courtesy: National Oilwell Varco Rig Systems

4. API RP 17B, "Recommended Pratice for Flexible Pipe", $4^{\text {th }}$ Ed.

5. P. O’Brien, E. Meldrum, C. Overton, J. Picksley, K. Anderson, I. MacLeod, “Outcomes from the SureFlex Joint Industry Project - An International Initiative on Flexible Pipe Integrity Assurance", Offshore Technology Conference, 2011

6. 4 Subsea, "Un-bonded Flexible Risers - Recent Field Experience and Actions for Increased Robustness", 2012

7. Directive 2013/30/EU on safety of offshore oil and gas operations, June 2013, Official Journal of the European Union

8. C. Marinho, C. Camerini, C. Maia, R Tadeu, and H Rocha H, "Gamma Ray System Operated by Robots for Underwater Pipeline Inspection", ECNDT 2006, Berlin, Sept 2006

9. J. R. McNab, B. Steel, "Computed radiography deployed by a subsea remotely operated vehicle", March 2008, Insight Journal, Vol. 50(3), pp. 138-145

10. P.I. Nicholson, "Development of an underwater radiography system for the in-situ inspection of flexible risers", Proceedings: 7th International QRM Conference, Quality Reliability and Maintenance, Coxmoor Publishing Company, 2010, p112-123, ISBN: 978 1-9-1892-34-5

11. L. Tomasz, "Pipe crawling underwater X-ray machines find leaks before they happen", Anti-Corrosion Methods and Materials, Vol. 61 Issue: 3, 2014, https://doi.org/10.1108/ACMM.12861caa.012 